\title{
Bio-inspired model of ground temperature behavior on the horizontal geothermal exchanger of an installation based on a heat pump
}

\author{
J.L. Casteleiro-Roca ${ }^{1}$, J.L. Calvo-Rolle ${ }^{1}$, M.C. Meizoso-Lopez ${ }^{1}$, A. \\ Piñón-Pazos ${ }^{1}$, B.A. Rodríguez-Gómez ${ }^{1}$ \\ ${ }^{a}$ Departamento de Ingeniería Industrial, University of A Coruña, A Coruña, Spain
}

\begin{abstract}
Nowadays the Heat Pump is one of the best systems to warm a building with a good performance. Usually, with the aim to increase the efficiency, a geothermal heat exchanger is added to the installation. This component shows a disturbing effect on the ground where it is placed. On this research a bio-inspired system was developed to test the ground temperature behavior where there is a heat exchanger. The novel approach has been implemented and tested under a real dataset. One year of temperature measurements were recorded. The final approach is based on clustering and regression techniques. Then, the model was validated and tested with a dataset from a real installation with a good performance.
\end{abstract}

Keywords: Local models, Clustering, SOM, MLP, SVM, Heat exchanger, Geothermal energy

\section{Introduction}

The heat systems in buildings based on a Heat Pump provide heat into a house by taking it out from a source [1]. The warmth can be obtained from any source, whether it is cold or hot [2]. If it is the second case, then it is possible to achieve higher efficiency [3]. Usually, the ground is a source for the Heat Pump and, the heat exchangers topology can be vertical

\footnotetext{
Email addresses: jose.luis.casteleiro@udc.es (J.L. Casteleiro-Roca), jlcalvo@udc.es (J.L. Calvo-Rolle), mmeizoso@udc.es (M.C. Meizoso-Lopez), andres.pinon@udc.es (A. Piñón-Pazos), benigno@udc.es (B.A. Rodríguez-Gómez) 
or horizontal $[1,4]$. The horizontal exchanger is cheaper than the vertical configuration [4]; however, this configuration is less effective. Due to this reason, frequently, installers place the exchanger more deeply in the ground to increase the efficiency [5].

Both configurations of heat exchangers present certain drawbacks, but the horizontal is more problematic than the other one, because among others, regardless of the depth, the exchanger is closer to the ground surface $[6,7]$. Due to the proximity to the surface, the meteorological conditions affect the exchanger and the efficiency could be lower [8,9].

The horizontal heat exchanger configuration usually is confined to a small space. Then, it is possible that the exchanger has an influence over the ground. If this happens, the temperature behavior of the ground could be different due to the influence of the exchanger [10]. When this phenomena happens, the heat system could show perceptible changes in behavior, and the non-linear behavior of the system increases [6]. Due to this fact, in this research, to determine the ground temperature behavior, a bio-inspired system has been developed based on local models. The aim of the study is to obtain a model to predict the ground performance that could allow to create, modify or improve the control algorithms of the system.

It is possible to divide the design of the prediction models in two methods [11]:

- Global models: only one model is generated based on all training data. The main aim is to minimize the error for all input patterns with the same model. Different techniques can be used to generate the model: Multi-Layer Perceptron (Artificial Neural Networks, ANN) [12, 13, 14], or Polynomial Regression [15] for instance.

- Local models: all the dataset is divided into subsets (clusters), depending on the features of the input data. The clustering algorithms are often used with this purpose like K-means or Self-Organizing Map (SOM) $[16,17]$. The first local models approaches were developed by [18] and [19], and were considered promising techniques in the field of time series prediction.

The combination of the local models with different regression techniques allows to obtain very satisfactory global results. When global models topologies were used, the results were poorer than with local models. A system of 
unsupervised learning SOM and K-means have been applied for the clustering phase. This bio-inspired model was created with a real dataset of a year of operation, then all four seasons, with all weather conditions, were taken into account.

This paper is organized as follows. It begins with a brief description of the case of study followed by an explanation of the bio-inspired model approach to describe the ground temperature behavior, and the dataset conditioning to obtain the best configuration through local models. In the next section, results are presented, and finally the conclusions and future works are shown.

\section{Case of study}

The model has been obtained to study the ground temperature behavior where a geothermal heat exchanger is placed. The heat exchanger is part of the Heat Pump installation within a real bioclimatic house. The system is described in the following sections.

\subsection{Sotavento bioclimatic house}

Sotavento bioclimatic house is a project of demonstrative bioclimatic house of Sotavento Galicia Foundation. The Foundation was created to study renewable energy and energy saving. It is located within the 'Parque eólico experimental de Sotavento', between the councils of Xermade (Lugo) and Monfero (A Coruña), in the autonomous community of Galicia (Spain). An external view of the bioclimatic house is shown in figure 1.

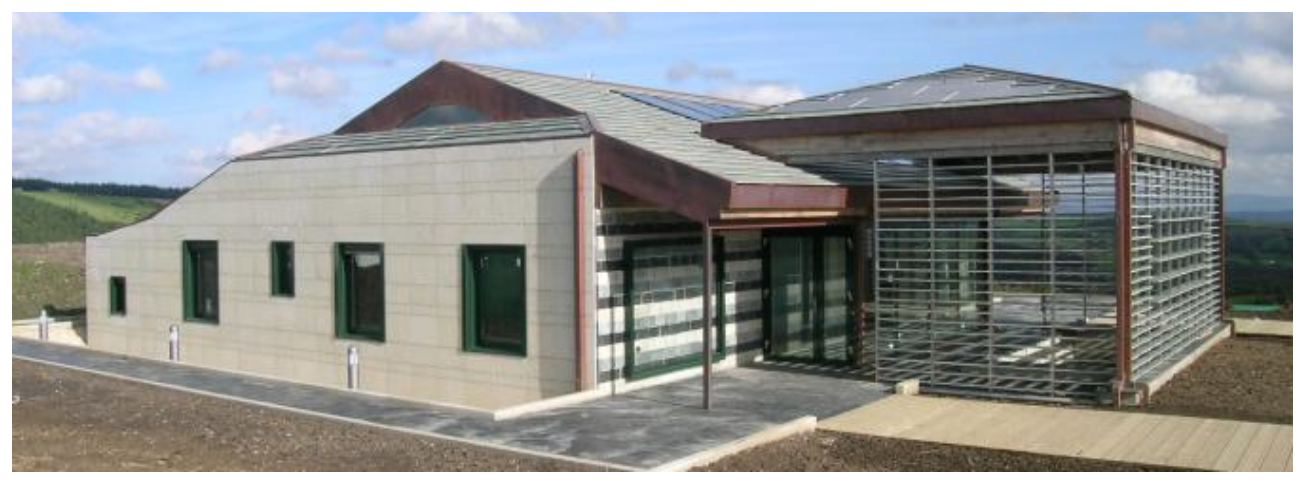

Figure 1: External view of the bioclimatic house 


\subsection{Installations of the bioclimatic house}

The bioclimatic house uses several sources of renewable energy for its facilities. The distribution of different thermal energy source is shown on figure 2. The whole thermal system, that includes the Domestic Hot Water (DHW) and the heating system, is powered by renewable sources, like Solar, Biomass and Geothermal.

For this research, we focus only on the thermal system, and inside this, only on a part of the geothermal installation.

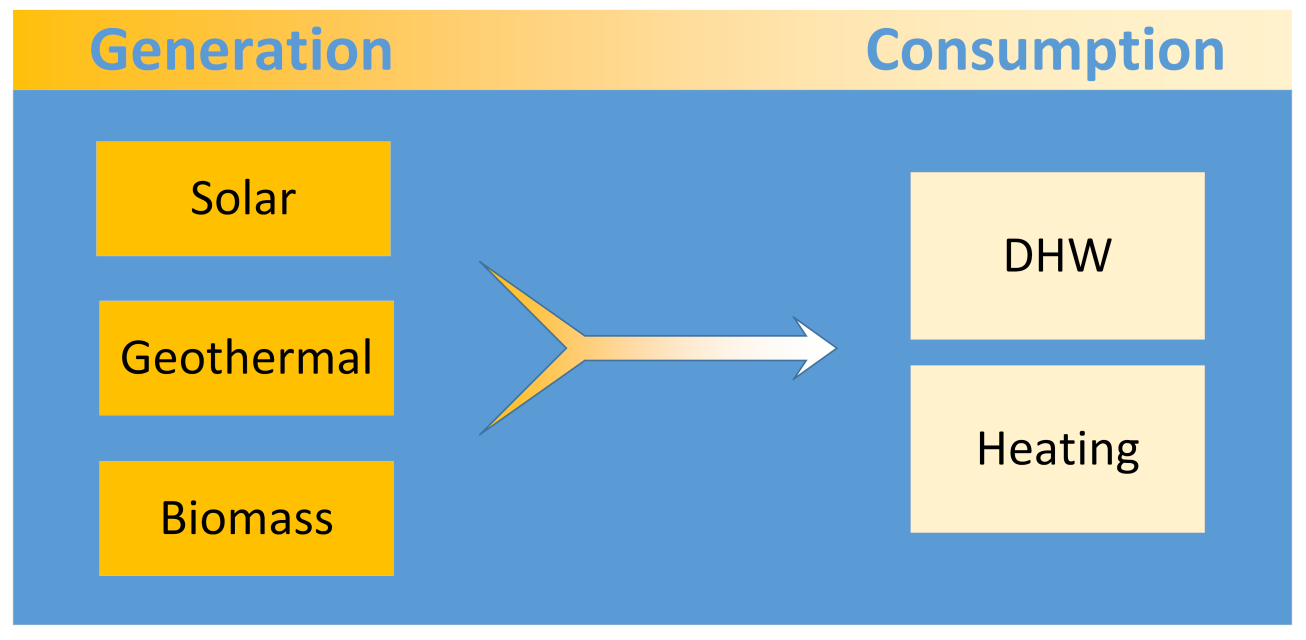

Figure 2: Thermal installations of the house

\subsection{Description of the thermal installation}

The different components of the thermal installation are shown in figure 3. It is possible to see that the installation is divided into 3 functional groups.

Generation. Three renewable energy sources are part of the generation group:

- Solar thermal system (1): It heats a fluid that flows inside panels, that capture energy from the solar radiation. The fluid goes to the accumulation zone, where it heats the water stored inside the solar accumulator. It is necessary to install this accumulator, because the fluid inside the panels is not water, it is ethylenglycol to prevent boiling in panels. 


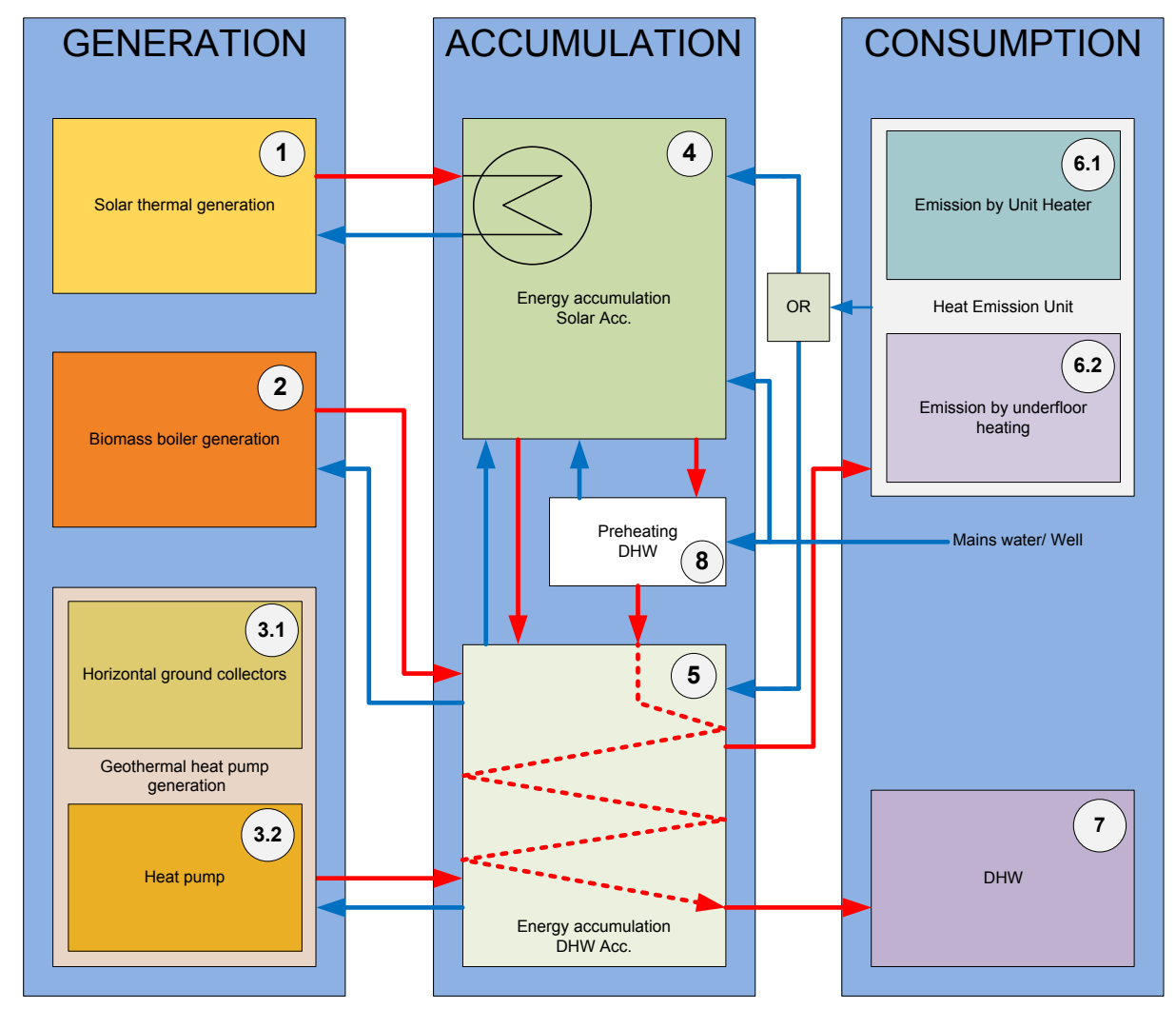

Figure 3: Block diagram of the thermal systems installed in the bioclimatic house

- Biomass boiler system (2): A biomass boiler, with a yield of pellets of $90 \%$, provides hot water directly to the inertial accumulator to ensure a temperature inside of around $63^{\circ} \mathrm{C}$.

- Geothermal system (3.1 and 3.2): The system consists in a combination of a Heat Pump and a horizontal exchanger. The exchanger was installed at 2 meters deep from the ground surface, and has 5 loops of 100 meters each one. The warm water from the Heat Pump is driven directly to the inertial accumulator as the biomass boiler.

Accumulation. This group has two accumulators, one specific for the solar energy (4), and another to supply the consumption equipment in the house (5). As it is possible that the temperature in this accumulator is less 
than the consumers desire, a preheating component (8) is installed in this section.

Consumption. The consumption is divided into two different uses: for warming the house (6.1 and 6.2) and DHW (7). Both uses are supplied through the inertial accumulator. The heat system is able to maintain the temperature inside the house between $18^{\circ} \mathrm{C}$ and $22^{\circ} \mathrm{C}$, and the DHW system was designed to supply a maximum of 240 liters per day, according to the Spanish Technical Building Code [20].

\subsection{Geothermal system under study}

This section gives a description of the real geothermal system and its components.

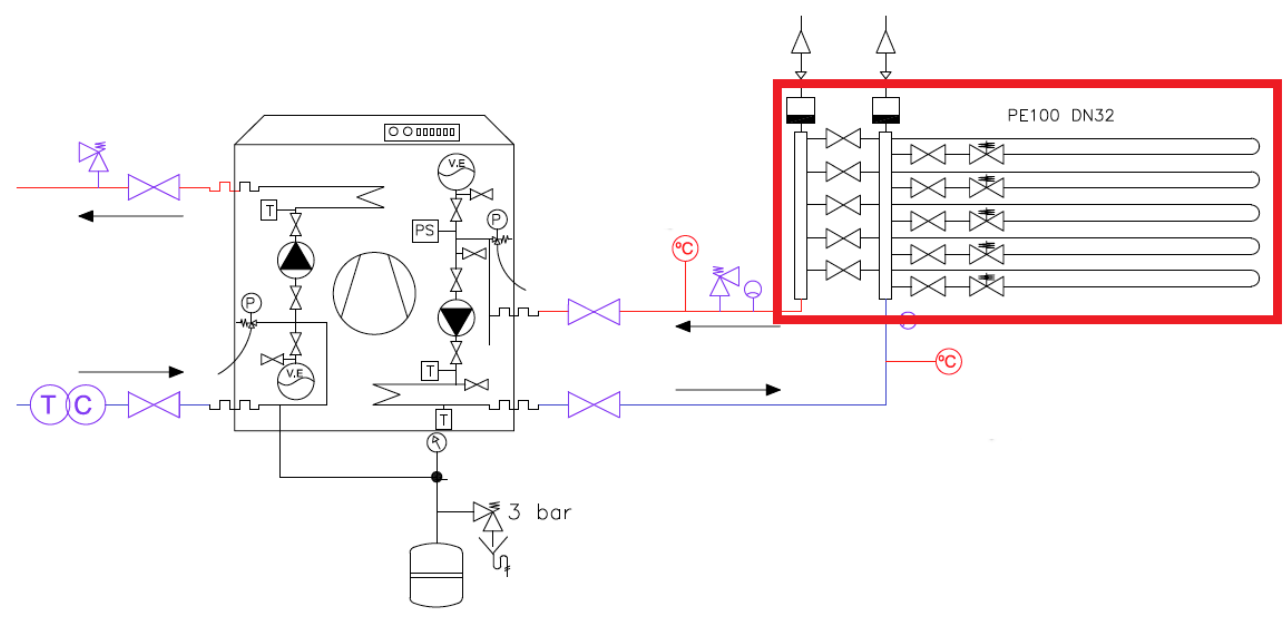

Figure 4: Heat Pump and horizontal exchanger layout

System description. The Heat Pump is a MAMY Genius - $10.3 \mathrm{~kW}$ with a horizontal heat exchanger as is shown in figure 4.

This study is only focused on part of the horizontal exchanger, framed in figure 4. This is the primary circuit of the Heat Pump, the secondary circuit, the output, is connected to the inertial accumulator.

Geothermal exchanger. The horizontal exchanger has five different loops. This configuration allows the isolation, of part of the exchanger, in case of failures. The exchanger has another four loops with eight temperature sensors each. These sensor loops were installed to study the ground temperature behavior. 
Under the ground, the exchanger loops are installed in one layer, and the sensor loops are above, with a similar distribution.

\section{Bio-inspired model approach}

The bio-inspired model approach is shown in figures 5 and 6 . Figure 5 shows the first step where the dataset is conditioned and the clusters are created. The figure 6 shows, in a graphical way, how the different regression models are obtained from the clusters achieved above. In the next subsections the procedure is explained in a detailed form.

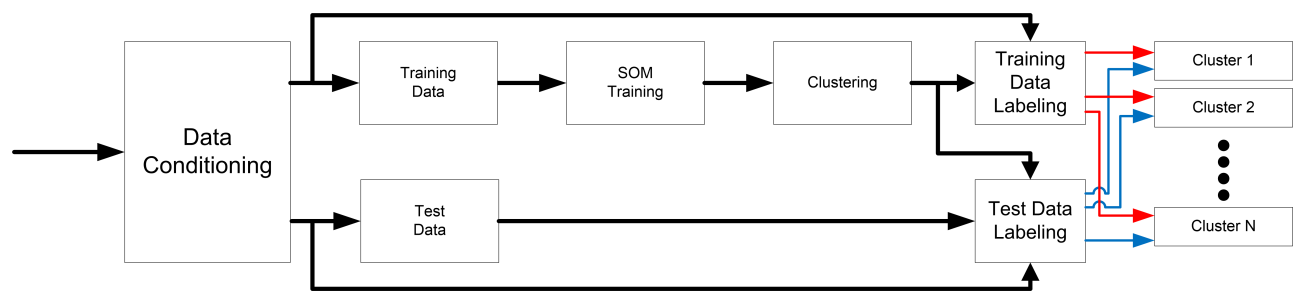

Figure 5: Dataset conditioning and cluster creation

\subsection{Obtaining and conditioning dataset}

The dataset used in this research is a complete set of one year temperature samples, obtained with a sample rate of 10 minutes. In figure 7 it is possible to see the ground temperature measured by a sensor placed out of the influence of the horizontal exchanger. That is the temperature modeling. As can be shown in this figure, there are some failures on the data acquisition. Then, the dataset was manually filtered to discard the erroneous data, and finally samples were reduced from 52705 to 52699 .

The dataset are the temperatures measured by eight of the sensors in the horizontal exchanger. Figure 8 shows the schema of the sensors approximate distribution along the heat exchanger, and the eight mentioned above are labeled.

\subsection{Techniques considered to create the model}

This section describes the techniques used to create the bio-inspired model. 

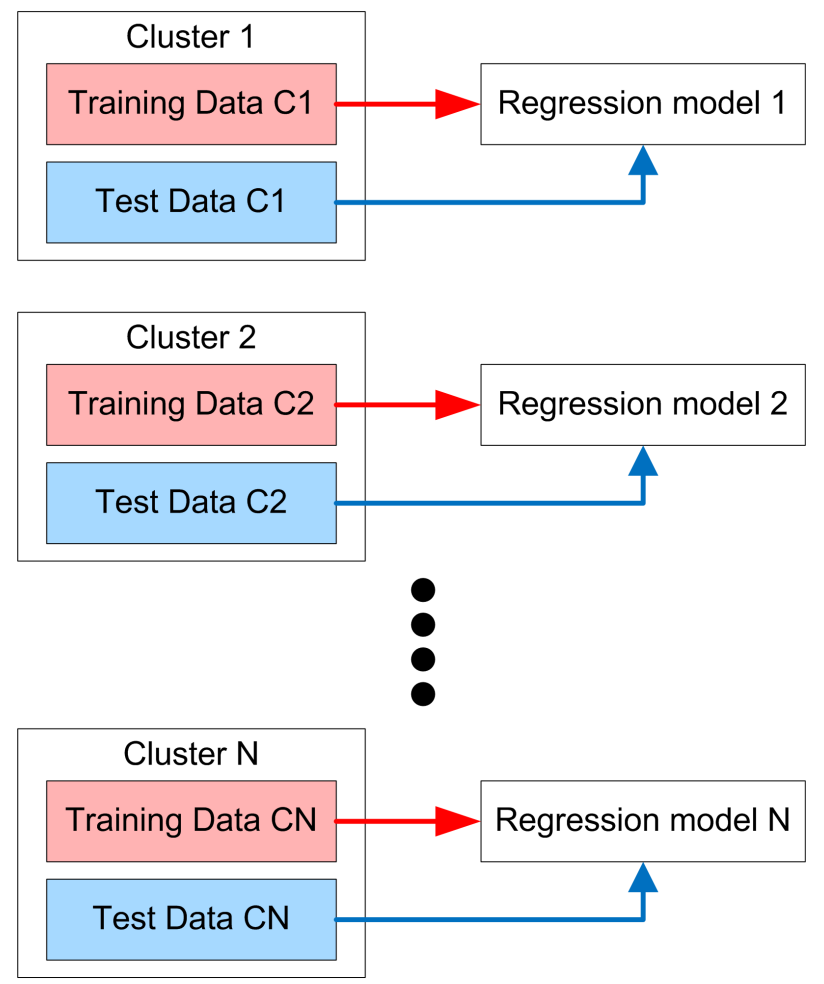

Figure 6: Obtaining regression models procedure

\subsubsection{Self-Organizing Map (SOM)}

SOM [21] was developed to visualize the data structure on a low dimensional display. It is also based on the use of unsupervised learning $[22,23]$. It consists of an array of nodes, with all nodes connected to the $N$ inputs by an $N$-dimensional weight vector. The self-organization process is implemented as an iterative on-line algorithm, although a batch version also exists. An input vector $(x)$ is presented to the network and the node of the network in which the weights $\left(W_{i}\right)$ are closest (euclidean distance) to $x$, is chosen by equation 1.

$$
c=\arg \min _{i}\left(\left\|x-W_{i}\right\|\right) .
$$

The weights of the winning node and the nodes close to it are then updated to move closer to the input vector. There is also a learning rate parameter that usually decreases as the training process progresses. The weight update rule for inputs is defined in equation 2 . 


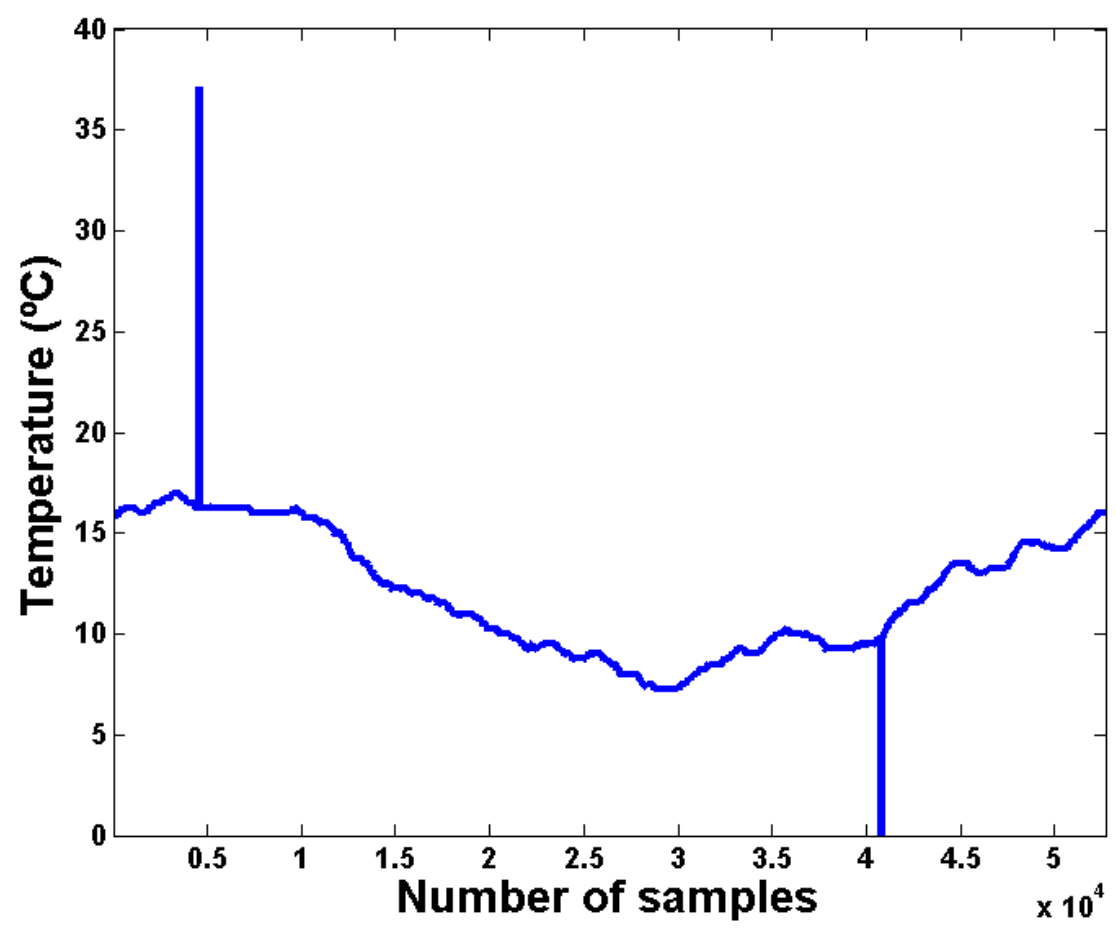

Figure 7: Ground temperature, pure data

$$
\Delta W_{i}=\eta(t) h_{c i}\left[x-W_{i}\right], \quad \forall i \in N^{(c)} .
$$

where, $W_{i}$ is the weight vector associated with neuron $i, x$ is the input vector, $h$ is the neighbourhood function (that depends on a neighbourhood radious), and $\eta(t)$ is the learning rate of the algorithm; $c$ represents the winning node.

After the training process, the SOM is expected to capture the inherent geometry of the process data. Then, it is possible to display the relationships between the input samples in 2D map. This information allows to obtain an initial idea of the number of clusters with a visual inspection of the map.

\subsubsection{Data Clustering. The K-means algorithm}

Clustering is an unsupervised technique of data grouping where similarity is measured $[24,25]$. Clustering algorithms try to organize unlabeled feature vectors into clusters or groups, such that, samples within a cluster are similar 


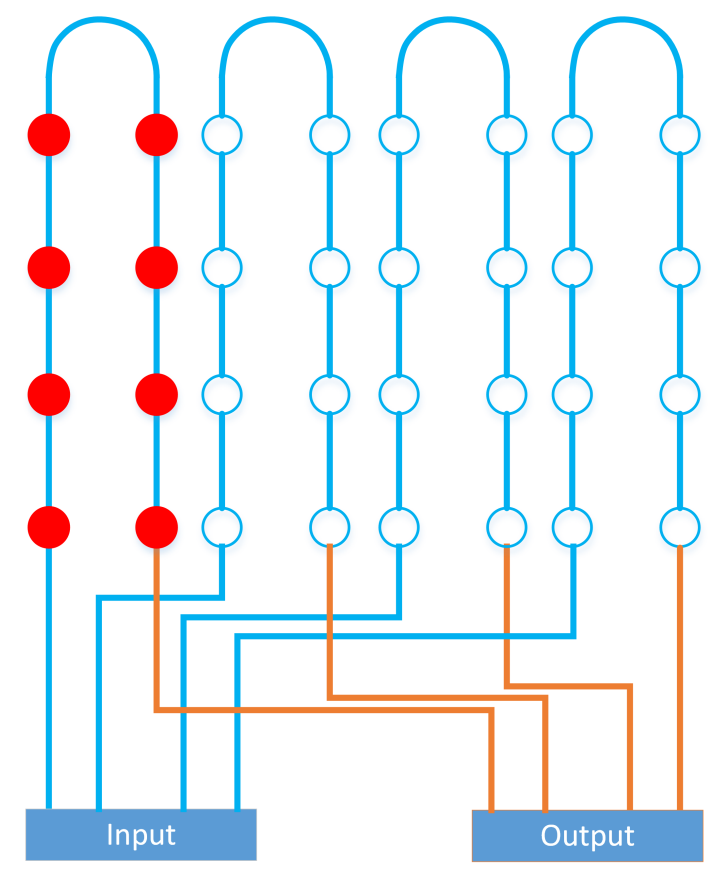

Figure 8: Horizontal exchanger's sensors layout

to each other [26]. A lot of clustering algorithms have been developed and new clustering algorithms continue to appear. Nevertheless, most of them are based on two common clustering methods: iterative square-error partitional clustering and agglomerative hierarchical clustering [27, 28, 29]. Partitional clustering algorithms divide a data set into a number of clusters, by trying to minimize several principles or error function. The quantity of clusters is typically predefined, but it can also be part of the error function [30, 31]. The vast majority of partitional clustering algorithms are based on the squareerror criterion. The general objective is to obtain that partition which, for a fixed number of clusters, reduces the square-error. K-means algorithm is a commonly used partitional clustering algorithm with square-error criterion, which minimizes error function 3.

$$
e=\sum_{k=1}^{C} \sum_{x \in Q_{k}}\left\|x-c_{k}\right\|^{2}
$$

The final clustering will depend on the initial cluster centers and on the value of $\mathrm{K}$. Choosing $\mathrm{K}$ value is the most critical thing because it requires 
certain prior knowledge of the number of clusters present in the data, which is highly doubtful. The K-means partitional clustering algorithm is computationally effective and works well if the clusters are close, hyperspherical in shape and well-separated in the hyperspace. The algorithm is able to detect hyper ellipsoidal-shaped clusters [29].

\subsubsection{Artificial Neural Networks (ANN). Multi-Layer Perceptron (MLP)}

A multilayer perceptron is a feedforward artificial neural network [32, 33]. It is one of the most typical ANNs due to its robustness and relatively simple structure. However the ANN architecture must be well selected to obtain good results. The MLP is composed by one input layer, one or more hidden layers and one output layer.

Each layer is made of neurons, with a specific activation function. In a usual configuration, all neurons from a layer have the same activation function. This function could be: Step, Linear, Log-sigmoid or Tan-sigmoid. Equation 4 shows the Tan-sigmoid function. All layers in MLP have weighted connections between neurons of each layer.

$$
F(t)=\frac{e^{t}-e^{-t}}{e^{t}+e^{-t}}
$$

It is possible to define the output of a MLP as shown in equation 5 [34].

$$
f_{\theta}(x)=\beta+\sum_{i=1}^{k} a_{i} \phi\left(w_{i}^{T} x+b_{i}\right)
$$

where:

$x=(x(1), \ldots, x(d))^{T} \in \Re^{d}$ is the vector of inputs

$k$ is the number of hidden layers

$\phi$ is a bounded transfer function

$\theta=\left(\beta, a_{1}, \ldots, a_{k}, b_{1}, \ldots, b_{k}, w_{11}, \ldots, w_{k d}\right)$ is the parameter vector of the model $w_{i}=\left(w_{i 1}, \ldots, w_{i d}\right)^{T} \in \Re^{d}$ is the parameter vector for the hidden unit $i$

\subsubsection{Polynomial regression}

Generally, a polynomial regression model [35, 36, 37] may also be defined as a linear summation of basis functions. The number of basis functions depends on the number of inputs of the model, and the degree of the polynomial used. With a degree 1 , the linear summation could be defined as the 
one shown in equation 6 . The model becomes more complex as the degree increases (equation 7 shows a second degree polynomial for a model).

$$
\begin{gathered}
F(x)=a_{0}+a_{1} x_{1}+a_{2} x_{2} \\
F(x)=a_{0}+a_{1} x_{1}+a_{2} x_{2}+a_{3} x_{1} x_{2}+a_{4} x_{1}^{2}+a_{5} x_{2}^{2}
\end{gathered}
$$

3.2.5. Support Vector Regression (SVR), Least Square Support Vector Regression (LS-SVR)

Support Vector Regression is a modification of the algorithm of the Support Vector Machines (SVM) for classification. In SVR the basic idea is to map the data into a high-dimensional feature space $\mathrm{F}$ via a non linear mapping and apply linear regression in this space [38, 39].

Least Square formulation of SVM, is called LS-SVM. The approximation of the solution is obtained by solving a system of linear equations, and it is comparable to SVM in terms of generalization performance [40, 41]. The application of LS-SVM to regression is known as LS-SVR (Least Square Support Vector Regression) [42, 43]. In LS-SVR, the insensitive loss function is replaced by a classical squared loss function, which constructs the Lagragian by solving a linear KarushKuhn-Tucker KKT (equation 8).

$$
\left[\begin{array}{cc}
0 & I_{n}^{T} \\
I_{n} & K+\gamma_{-1} I
\end{array}\right]\left[\begin{array}{l}
b_{0} \\
b
\end{array}\right]=\left[\begin{array}{l}
0 \\
y
\end{array}\right]
$$

where $I_{n}$ is a vector of $n$ ones, $T$ means transpose of a matrix or vector, $\gamma$ a weight vector, $b$ regression vector and $b_{0}$ is the model offset. In LS-SVR, only two parameters $(\gamma, \sigma)$ are needed. Where, $\sigma$ is the width of the used kernel [44]

\subsection{Model Test.}

The dataset was split as usual, into two groups of samples (2/3 for training and $1 / 3$ for testing) and MSE (Mean Square-Error) was calculated for each model at the testing step.

\subsection{Model Selection.}

On this step, the best model for each cluster was selected according to the MSE. The MSE of the selected models is compared to the MSE of each global regression model. 


\section{Results}

Different tests were made to choose the best model for the system. At first, it was necesary to analyze the number of clusters in the dataset. After the number of clusters was chosen, the dataset was divided in clusters using K-means algorithm. For each cluster, different regression techniques were tested to choose the best one.

\subsection{SOM analysis}

Figure 9 shows the neighbor distance map after an analysis with the SOM technique. The clusters are defined in the figure among the different sections of color in it. The neighbor distance defines the different zones of neurons in the SOM structure. Dark lines are the borders between clusters, while light area on the map corresponds to cluster. In figure 9 it is possible to distinguish that there are some clusters, but it is really difficult to decide the optimal number of them for this dataset.

Figure 10 shows the component planes for each input of the SOM net (the eight temperature sensors). The different planes represent the reaction of the SOM net to the specific input. In this research, 'Input 2' and 'Input 3' have a similar reaction, and this means that the two inputs are correlated with one another. It is possible to extract the same conclusion for the 'Input 5' and 'Input 6', but in this case, the correlation is not very strong.

In this research, the optimal number of clusters was decided after testing between 3 to 7 clusters.

The best result has been achieved with the next parameters:

- SOM-net dimension of 20x20 neurons

- Kernel: Gaussian

- Training algorithm: Unsupervised batch training

- Initialization algorithm: Random

- Learning rate: Sequential

- Epoch number: between 200 and 300 


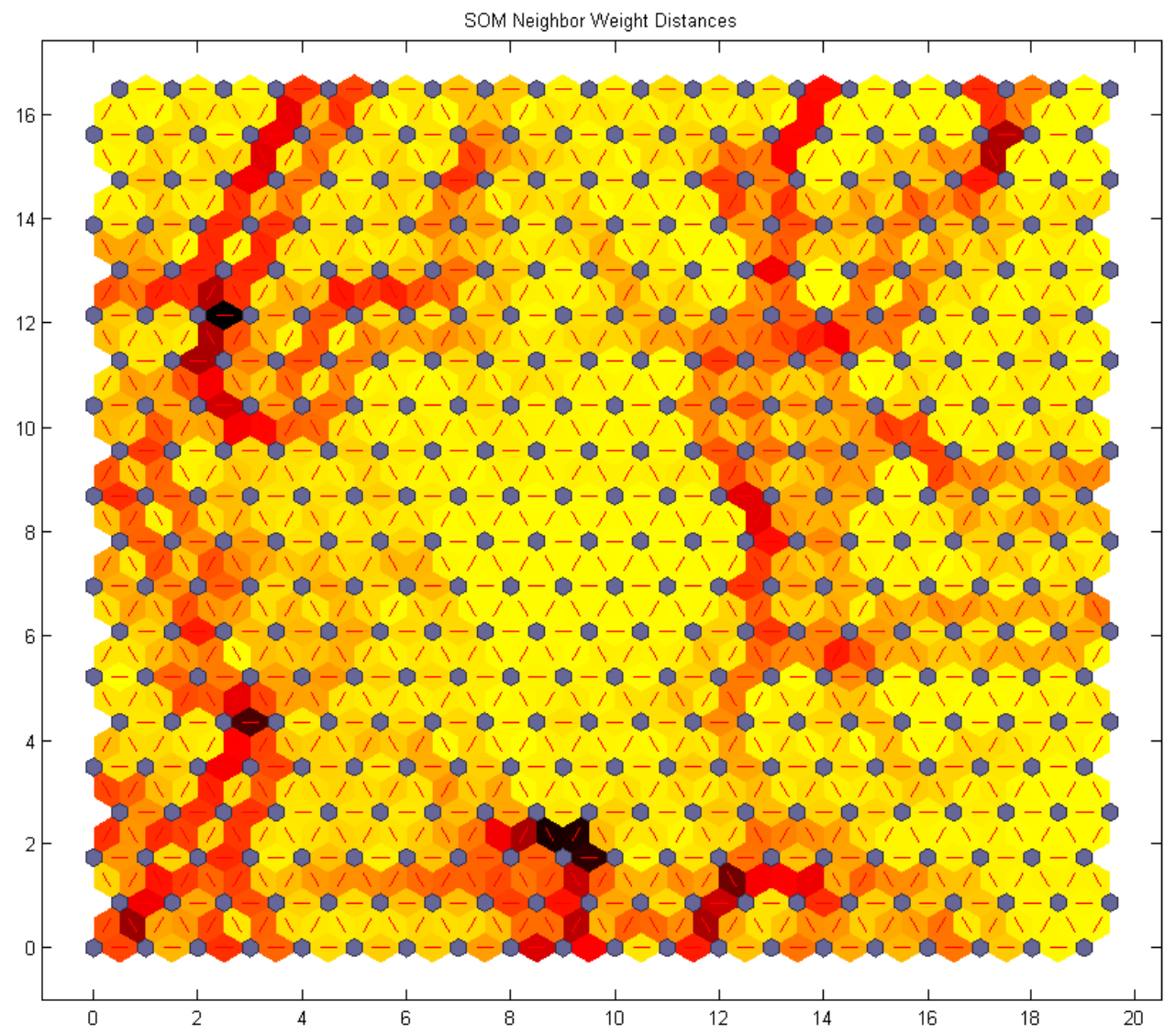

Figure 9: SOM results, Neighbor distance

\subsection{Regression analysis}

As was presented, the number of clusters was decided after testing different numbers of them. In table 1 , it is possible to see the number of samples assigned to each cluster for training and testing the model achieved.

For each cluster, a model was trained following the explained regression techniques. A summary of the tests made for a 4 cluster division is presented in table 2. The accuracy for each model is evaluated in MSE values. The best results were always achieved with ANN-MLPs with only one hidden layer.

Table 3 shows the increase of accuracy achieved by using local models instead of a global model.

The graphic comparative of MSE against the number of clusters is shown in figure 11, and it is clear that the results are very similar in all cases, but 

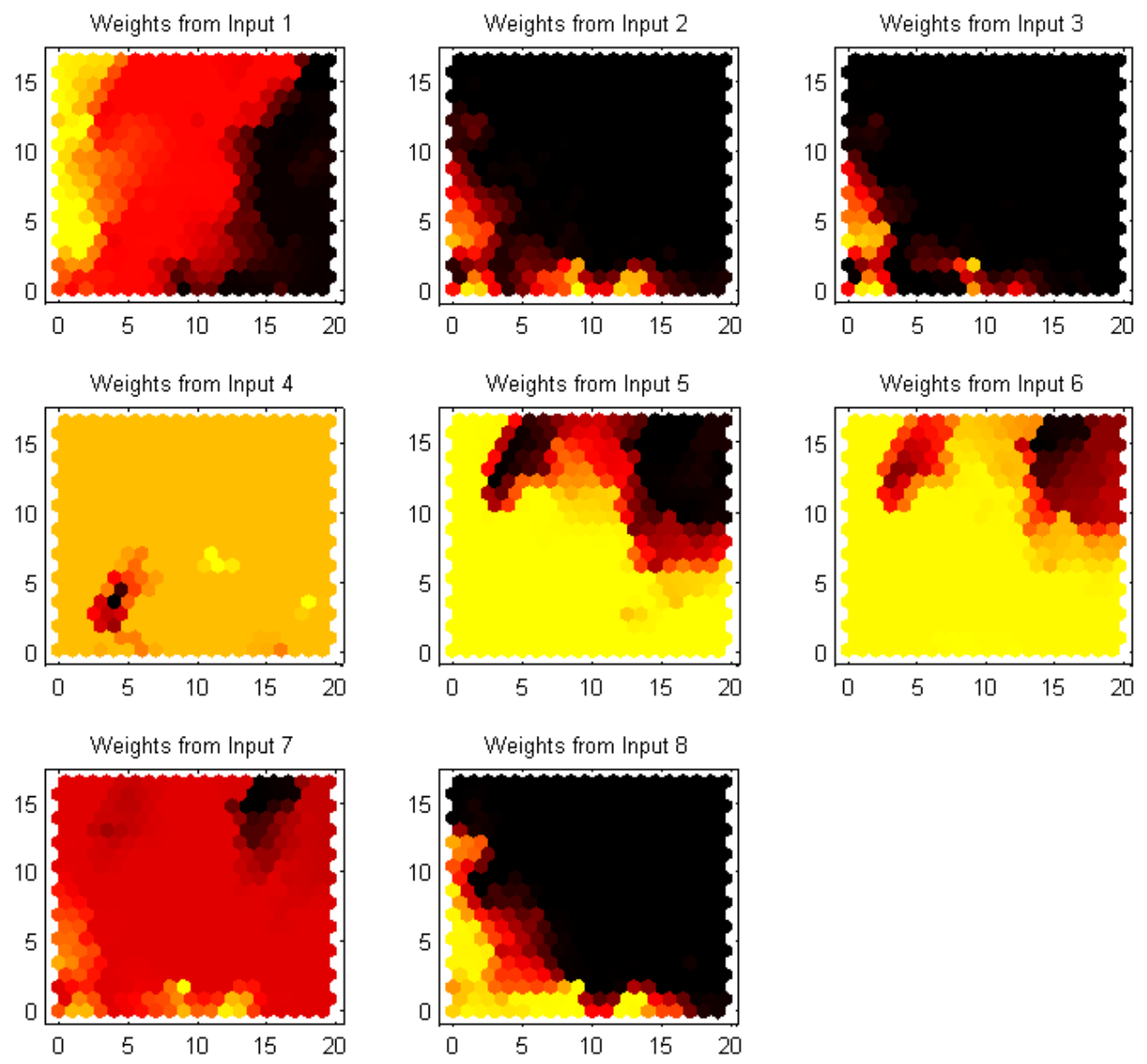

Figure 10: SOM results, Input planes

with 4 clusters is the way to achieve the best results.

Depending on the cluster, the best results were obtained by using a number of neurons between 8 to 15 with a Tan-sigmoidal transfer function for the hidden layer, and the output layer transfer function was linear.

By using LS-SVRs, the best approximation is achieved, also depending on the cluster, on the next ranges: $\gamma=2.9623 E 7-2.4528 E 7 \sigma^{2}=1.73248-2.20117$.

The best approximation has been obtained by using degree 6 . The degrees tested range is from 3 to 9 . 


\begin{tabular}{|c|c|c|c|c|}
\hline $\begin{array}{l}\text { Clusters } \\
\text { quantity }\end{array}$ & $\begin{array}{l}\text { cluster } \\
\text { number }\end{array}$ & Train & Test & Total \\
\hline \multicolumn{2}{|c|}{ No clusters } & 35133 & $\overline{c 17566}$ & $\bar{~} 52699$ \\
\hline \multirow{3}{*}{3 Clusters } & 1 & 10244 & 5077 & 15321 \\
\hline & 2 & 14714 & 7369 & 22083 \\
\hline & 3 & 10175 & 5120 & 15295 \\
\hline \multirow{4}{*}{4 Clusters } & 1 & 9714 & 4874 & 14588 \\
\hline & 2 & 6507 & 3257 & 9764 \\
\hline & 3 & 12433 & 6229 & 18662 \\
\hline & 4 & 6479 & 3206 & 9685 \\
\hline \multirow{5}{*}{5 Clusters } & 1 & 3443 & 1722 & 5165 \\
\hline & 2 & 6441 & 3184 & 9625 \\
\hline & 3 & 5954 & 2981 & 8935 \\
\hline & 4 & 12422 & 6227 & 18649 \\
\hline & 5 & 6873 & 3452 & 10325 \\
\hline \multirow{6}{*}{6 Clusters } & $\overline{c 1}$ & 6870 & 3450 & 10320 \\
\hline & 2 & 3430 & 1646 & 5076 \\
\hline & 3 & 4842 & 2557 & 7399 \\
\hline & 4 & 10536 & 5286 & 15822 \\
\hline & 5 & 4473 & 2217 & 6690 \\
\hline & 6 & 4982 & 2410 & 7392 \\
\hline \multirow{7}{*}{7 Clusters } & 1 & 4660 & 2454 & 7114 \\
\hline & 2 & 7066 & 3548 & 10614 \\
\hline & 3 & 3694 & 1891 & 5585 \\
\hline & 4 & 4980 & 2405 & 7385 \\
\hline & 5 & 3430 & 1646 & 5076 \\
\hline & 6 & 6870 & 3450 & 10320 \\
\hline & 7 & 4433 & 2172 & 6605 \\
\hline
\end{tabular}

Table 1: Number of samples data for training and testing

\section{Conclusions and future works}

This research shows a bio-inspired model. It has been developed to test the ground temperature behavior on the horizontal geothermal exchanger of an installation based on a heat pump. With the aim to obtain a good fitness the dataset is from an entire year. The model has been presented, analyzed 


\begin{tabular}{|l|c|c|c|c|c|}
\hline & Train & Test & \multicolumn{3}{|c|}{ MSE } \\
\cline { 4 - 6 } & Samples & Samples & ANN-MLP & Polynomial & LS-SVR \\
\hline Cluster 1 & 9714 & 4874 & 0.00632 & 0.00882 & 0.00678 \\
\hline Cluster 2 & 6507 & 3257 & 0.00493 & 0.00926 & 0.00513 \\
\hline Cluster 3 & 12433 & 6229 & 0.00613 & 0.01034 & 0.00671 \\
\hline Cluster 4 & 6479 & 3206 & 0.00598 & 0.00974 & 0.00632 \\
\hline
\end{tabular}

Table 2: MSE for different regression techniques - 4 clusters

\begin{tabular}{|c|c|c|}
\hline \multicolumn{2}{|c|}{} & MSE \\
\hline \multicolumn{2}{|c|}{ Global model } & 0.0221 \\
\hline \multirow{4}{*}{ Local models } & 3 Clusters & 0.0076 \\
\cline { 2 - 3 } & 4 Clusters & 0.0059 \\
\cline { 2 - 3 } & 5 Clusters & 0.0069 \\
\cline { 2 - 3 } & 6 Clusters & 0.0063 \\
\cline { 2 - 3 } & 7 Clusters & 0.0121 \\
\hline
\end{tabular}

Table 3: MSE comparation

and compared with others.

The proposal is based on local models combined with intelligent techniques for regression. Taken into account the modeled system, which has a very non linear component, the main advantages of the achieved model are two: the high accuracy and the simplicity. The best result has been obtained with four clusters and, the artificial neural networks offer the best mean square error on regression. The other regression techniques give acceptable results.

Future works will be focused on other regression techniques and unsupervised learning to create clusters, with the objective to improve the achieved model. Also, the present approach will be applied to other models where the system has a higher non linear component. For the same building, the next works will be focused on optimization tasks to reduce the energy consumption without losing comfort.

\section{Acknowledgements}

We would like to thank the 'Instituto Enerxético de Galicia' (INEGA) and 'Parque Eólico Experimental de Sotavento' (Sotavento Foundation) for their technical support in this work. 


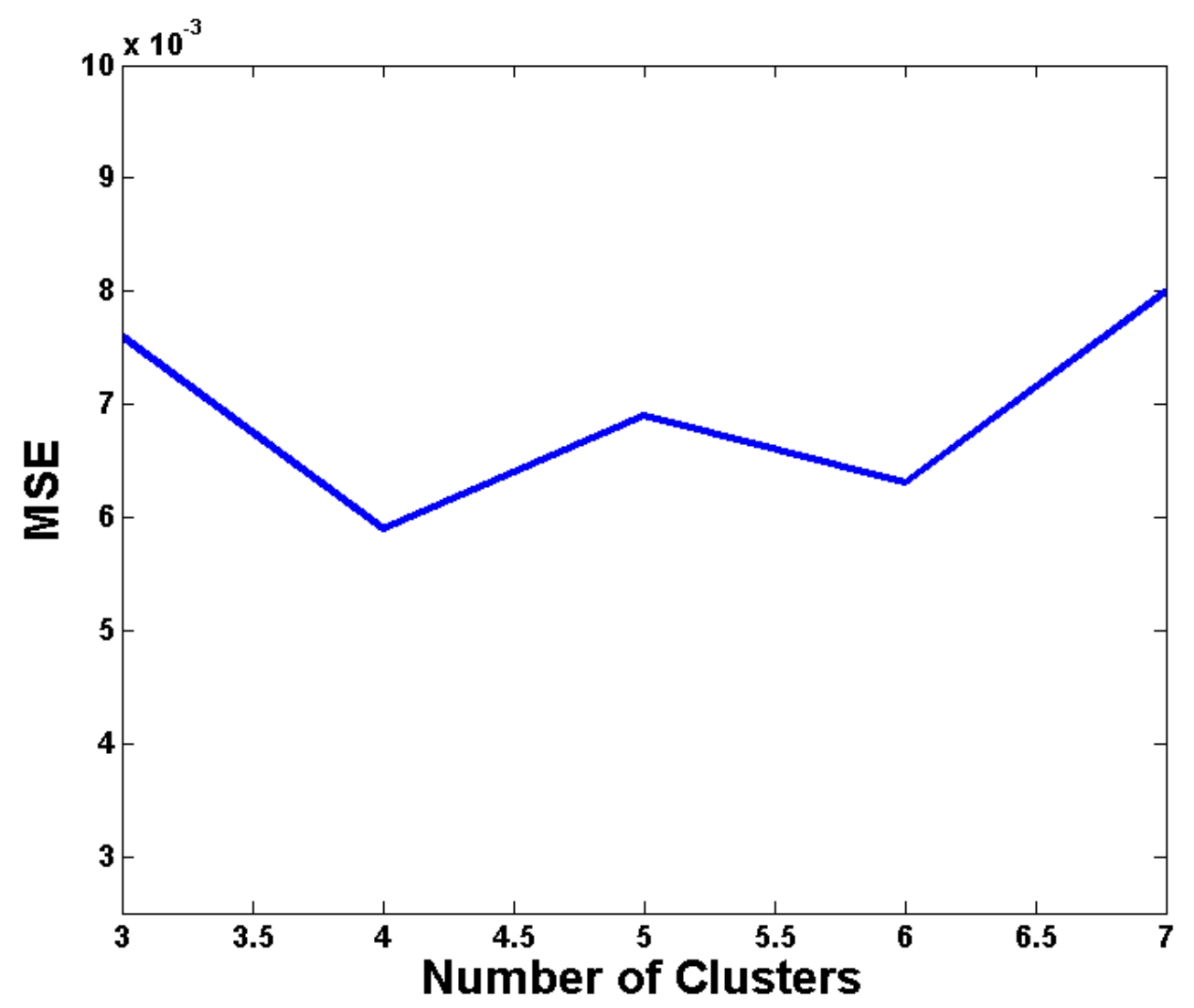

Figure 11: MSE against number of clusters

\section{References}

[1] B. Langley, Heat Pump technology, Prentice Hall PTR, 2002.

[2] C. Ugalde-Caballero, J. Antunez-Marin, Low temperature thermal micro energy harvester, Dyna 87 (6) (2012) 640-656. doi:http://dx.doi.org/10.6036/5006.

[3] H. Sauer, R. Howell, Heat Pump systems, Krieger Publishing Company, 1991.

[4] S. Kakaç, H. Liu, Heat exchangers: selection, rating, and thermal design, Designing for heat transfer, CRC Press, 2002. 
[5] A. Rezaei, E. Kolahdouz, G. Dargush, A. Weber, Ground source heat pump pipe performance with tire derived aggregate, International Journal of Heat and Mass Transfer 55 (11-12) (2012) 2844-2853. doi:http://dx.doi.org/10.1016/j.ijheatmasstransfer.2012.02.004.

[6] C. Lee, Effects of multiple ground layers on thermal response test analysis and ground-source heat pump simulation, Applied Energy $88 \quad$ (12) (2011) 4405-4410. doi:http://dx.doi.org/10.1016/j.apenergy.2011.05.023.

[7] B. Sanner, C. Karytsas, D. Mendrinos, L. Rybach, Current status of ground source heat pumps and underground thermal energy storage in europe, Geothermics 32 (4-6) (2003) 579-588. doi:http://dx.doi.org/10.1016/S0375-6505(03)00060-9.

[8] V. Tarnawski, W. Leong, T. Momose, Y. Hamada, Analysis of ground source heat pumps with horizontal ground heat exchangers for northern japan, Renewable Energy 34 (1) (2009) 127-134. doi:http://dx.doi.org/10.1016/j.renene.2008.03.026.

[9] A. Omer, Ground-source heat pumps systems and applications, Renewable and sustainable energy reviews 12 (2) (2008) 344-371. doi:http://dx.doi.org/10.1016/j.rser.2006.10.003.

[10] D. Banks, An introduction to thermogeology: ground source heating and cooling, Wiley, 2009.

[11] D. Martínez-Rego, O. Fontenla-Romero, A. Alonso-Betanzos, Efficiency of local models ensembles for time series prediction, Expert Syst. Appl. 38 (6) (2011) 6884-6894. doi:10.1016/j.eswa.2010.12.036.

[12] C. Bishop, Pattern recognition and machine learning (information science and statistics), Springer-Verlag New York, Inc., Secaucus, NJ, USA, 2006.

[13] J. Calvo-Rolle, J. Casteleiro-Roca, H. Quintián, M. Meizoso-Lopez, A hybrid intelligent system for PID controller using in a steel rolling process, Expert Systems with Applications 40 (13) (2013) 5188-5196. doi:http://dx.doi.org/10.1016/j.eswa.2013.03.013. 
[14] A. Alvarez-Huerta, R. Gonzalez-Miguelez, D. García-Metola, A. Noriega-Gonzalez, Drywell tempeture prediction of a nuclear power plant by means of artificial neural networks, Dyna 86 (4) (2011) 467-473. doi:http://dx.doi.org/10.6036/4025.

[15] E. Alpaydin, Introduction to Machine Learning, Adaptive computation and machine learning, MIT Press, 2004.

[16] A. Cherif, H. Cardot, R. Boné, SOM time series clustering and prediction with recurrent neural networks, Neurocomput. 74 (11) (2011) 1936-1944. doi:10.1016/j.neucom.2010.11.026.

[17] M. H. Ghaseminezhad, A. Karami, A novel self-organizing map (SOM) neural network for discrete groups of data clustering, Appl. Soft Comput. 11 (4) (2011) 3771-3778. doi:10.1016/j.asoc.2011.02.009.

[18] R. Jacobs, M. Jordan, S. Nowlan, G. Hinton, Adaptive mixtures of local experts, Neural Comput. 3 (1) (1991) 79-87. doi:10.1162/neco.1991.3.1.79.

[19] M. Jordan, R. Jacobs, Hierarchical mixtures of experts and the EM algorithm, Neural Comput. 6 (2) (1994) 181-214. doi:10.1162/neco.1994.6.2.181.

[20] R.D. 314/2006, 17 march, which approves the 'Código Técnico de la Edificación' (BOE 74, 2006).

[21] T. Kohonen, The self-organizing map, Proceedings of the IEEE 78 (9) (1990) 1464-1480. doi:10.1109/5.58325.

[22] T. Kohonen, E. Oja, O. Simula, A. Visa, J. Kangas, Engineering applications of the self-organizing map, Proceedings of the IEEE 84 (10) (1996) 1358-1384. doi:10.1109/5.537105.

[23] T. Kohonen, Exploration of very large databases by self-organizing maps, in: Neural Networks,1997., International Conference on, Vol. 1, 1997, pp. PL1-PL6 vol.1. doi:10.1109/ICNN.1997.611622.

[24] A. Qin, P. Suganthan, Enhanced neural gas network for prototypebased clustering, Pattern Recogn. 38 (8) (2005) 1275-1288. doi:10.1016/j.patcog.2004.12.007. 
[25] J. Ye, T. Xiong, Svm versus least squares svm, Journal of Machine Learning Research - Proceedings Track 2 (2007) 644-651.

[26] S. Kaski, J. Sinkkonen, A. Klami, Discriminative clustering, Neurocomputing $69 \quad$ (13) (2005) 18-41. doi:http://dx.doi.org/10.1016/j.neucom.2005.02.012.

[27] N. Pal, J. Biswas, Cluster validation using graph theoretic concepts, Pattern Recognition 30 (6) (1997) 847-857. doi:http://dx.doi.org/10.1016/S0031-3203(96)00127-6.

[28] A. Laha, N. Pal, Dynamic generation of prototypes with self-organizing feature maps for classifier design, Pattern Recognition 34 (2) (2001) 315-321. doi:http://dx.doi.org/10.1016/S0031-3203(99)00232-0.

[29] G. Şerban, A. Câmpan, Hierarchical adaptive clustering, Informatica 19 (1) (2008) 101-112.

[30] L. Garg, S. Mcclean, B. Meenan, P. Millard, Phase-type aurvival trees and mixed distribution survival trees for clustering patients' hospital length of stay, Informatica 22 (1) (2011) 57-72.

[31] K. Šutienè, D. Makackas, H. Pranevičius, Multistage k-means clustering for scenario tree construction, Informatica 21 (1) (2010) 123-138.

[32] P. Wasserman, Advanced methods in neural computing, 1st Edition, John Wiley \& Sons, Inc., New York, NY, USA, 1993.

[33] Z. Zeng, J. Wang, Advances in neural network research and applications, 1st Edition, Springer Publishing Company, Incorporated, 2010.

[34] J. Rynkiewicz, General bound of overfitting for MLP regression models, Neurocomputing 90 (0) (2012) 106-110. doi:http://dx.doi.org/10.1016/j.neucom.2011.11.028.

[35] R. Heiberger, E. Neuwirth, Polynomial regression, in: R Through Excel, Use R, Springer New York, 2009, pp. 269-284. doi:10.1007/978-1-44190052-4_11.

[36] X. Wu, Optimal designs for segmented polynomial regression models and web-based implementation of optimal design software, State University of New York at Stony Brook, Stony Brook, NY, USA, 2007. 
[37] Z. Zhang, S. C. Chan, On kernel selection of multivariate local polynomial modelling and its application to image smoothing and reconstruction, J. Signal Process. Syst. 64 (3) (2011) 361-374. doi:10.1007/s11265010-0495-4.

[38] N. Cristianini, J. Shawe-Taylor, An introduction to support Vector Machines and other kernel-based learning methods, Cambridge University Press, New York, NY, USA, 2000.

[39] I. Steinwart, A. Christmann, Support vector machines, 1st Edition, Springer Publishing Company, Incorporated, 2008.

[40] J. Suykens, J. Vandewalle, Least squares support vector machine slassifiers, Neural Processing Letters 9 (3) (1999) 293-300. doi:10.1023/A:1018628609742.

[41] R. Wang, A. Wang, Q. Song, Research on the alkalinity of sintering process based on LS-SVM algorithms, in: D. Jin, S. Lin (Eds.), Advances in Computer Science and Information Engineering, Vol. 168 of Advances in Intelligent and Soft Computing, Springer Berlin Heidelberg, 2012, pp. 449-454. doi:10.1007/978-3-642-30126-1_71.

[42] Y. Guo, X. Li, G. Bai, J. Ma, Time series prediction method based on LS-SVR with modified gaussian RBF, in: T. Huang, Z. Zeng, C. Li, C. Leung (Eds.), Neural Information Processing, Vol. 7664 of Lecture Notes in Computer Science, Springer Berlin Heidelberg, 2012, pp. 9-17. doi:10.1007/978-3-642-34481-7_2.

[43] L. Wang, J. Wu, Neural network ensemble model using PPR and LSSVR for stock market eorecasting, in: D.-S. Huang, Y. Gan, V. Bevilacqua, J. Figueroa (Eds.), Advanced Intelligent Computing, Vol. 6838 of Lecture Notes in Computer Science, Springer Berlin Heidelberg, 2012, pp. 1-8. doi:10.1007/978-3-642-24728-6_1.

[44] Y. Li, X. Shao, W. Cai, A consensus least squares support vector regression (LS-SVR) for analysis of near-infrared spectra of plant samples, Talanta 72 (1) (2007) 217-222. doi:http://dx.doi.org/10.1016/j.talanta.2006.10.022. 


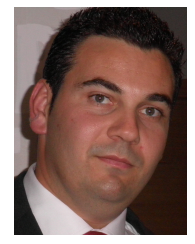

Jose Luis Casteleiro-Roca was born in A Coruna, Spain, in 1981. He received the B.S. from University of Coruna in 2003, the M.S. in Industrial Engineering from the University of Leon in 2012, and now he is a Ph.D student in the University of Coruna. He is a Technical Engineer in Spanish Navy, and he has been working in Ferrol's Missiles workshop since 2004. His main research interests have been centered in applying expert system technologies to the diagnosis and control systems and in intelligent systems for control engineering and optimization.

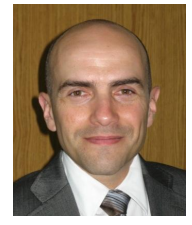

Jose Luis Calvo-Rolle was born in A Coruna, Spain, in 1974. He received the M.S. and Ph.D. degrees in Industrial Engineering from the University of Leon, Leon, Spain, in 2004, and 2007, respectively. He is Associate Professor of Automatic Control and the head of Industrial Engineering Department, Faculty of Engineering, University of A Coruna, Spain. His main research interests have been centered in applying expert system technology to the diagnosis and control systems and in intelligent training systems for control engineering, optimization and education.

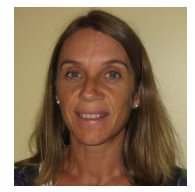

María del Carmen Meizoso-López was born in A Coruna, Spain, in 1967. She received the M.S. degree in Telecomunications Engineering from the University of Vigo, Pontevedra, Spain, in 1994 and the Ph.D. degree in Industrial Engineering from the University of A Coruna, Spain in 2012. She joined the Industrial Engineering Department at University of A Coruna, Spain as Associate Professor in 1994. She has led and participated in several research projects. Her main research interests include solar energy, temporal series of solar radiation.

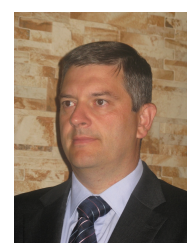

Andres Piñón-Pazos was born in A Coruna, Spain, in 1969. He received the M.S. degrees in Industrial Engineering from the University of Coruna, Coruna, Spain, in 1998. He is Associate Professor in the Systems Engineering and Automatic Control area of Industrial Engineering Department, Faculty of Engineering, University of A Coruna, Spain. His main research is centered on sensors, electronic instrumentation and fieldbus sensors.

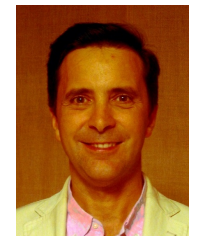

Benigno Antonio Rodríguez Gómez received his B.S. in Science (Physics) from the University of Santiago de Compostela, Spain, in 1988, and his $\mathrm{PhD}$ in Science (Environmental) from the University of A Coruna, Spain in 2010. $\mathrm{He}$ is Asociate Professor of Instrumentation and Control Systems at the Faculty of Nautical Science and Naval Machinery, University of A Coruna, Spain. His current specific interests include assessment of natural resources and systems modeling. 